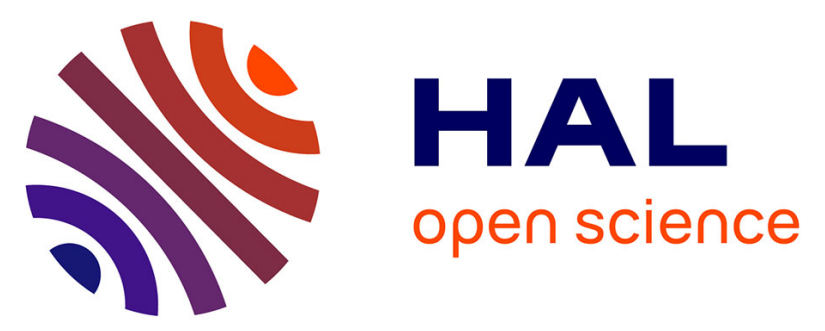

\title{
A new strategy for the synthesis of monomethylhydrazine using the Raschig process. 2: Continuous synthesis of stoichiometric monochloramine using the microreactor technology
}

Duc Minh Le, Anne-Julie Bougrine, Olivier Duclos, Véronique Pasquet, Henri Delalu

\section{To cite this version:}

Duc Minh Le, Anne-Julie Bougrine, Olivier Duclos, Véronique Pasquet, Henri Delalu. A new strategy for the synthesis of monomethylhydrazine using the Raschig process. 2: Continuous synthesis of stoichiometric monochloramine using the microreactor technology. Reaction kinetics, mechanisms and catalysis, 2020, 130(1), pp.17-34. 10.1007/s11144-020-01761-4 . hal-03018980

\author{
HAL Id: hal-03018980 \\ https://hal.science/hal-03018980
}

Submitted on 24 Nov 2020

HAL is a multi-disciplinary open access archive for the deposit and dissemination of scientific research documents, whether they are published or not. The documents may come from teaching and research institutions in France or abroad, or from public or private research centers.
L'archive ouverte pluridisciplinaire HAL, est destinée au dépôt et à la diffusion de documents scientifiques de niveau recherche, publiés ou non, émanant des établissements d'enseignement et de recherche français ou étrangers, des laboratoires publics ou privés. 


\section{Reaction Kinetics, Mechanisms and Catalysis}

\section{A new strategy for the synthesis of monomethylhydrazine using the Raschig process. 2 - Continuous synthesis of stoichiometric monochloramine using the microreactor technology --Manuscript Draft--}

Manuscript Number:

Full Title:

Article Type:

Corresponding Author:
REAC-D-20-00005R1

A new strategy for the synthesis of monomethylhydrazine using the Raschig process. 2 - Continuous synthesis of stoichiometric monochloramine using the microreactor technology

Full Paper

Véronique Pasquet

Laboratoire Hydrazines et Composés Energétiques Polyazotés

Villeurbanne, FRANCE

\section{Corresponding Author Secondary} Information:

Corresponding Author's Institution:

Laboratoire Hydrazines et Composés Energétiques Polyazotés

Corresponding Author's Secondary Institution:

First Author:

Duc Minh Le

First Author Secondary Information:

Order of Authors:

Duc Minh Le

Anne-Julie Bougrine

Olivier Duclos

Véronique Pasquet

Henri Delalu

Order of Authors Secondary Information:

Funding Information:

Abstract:

After having established in a previous paper that the synthesis of stoichiometric monochloramine is hazardous due to the high exothermicity of the reaction and the risk of decomposition of the solution from $36^{\circ} \mathrm{C}$, it appeared that the best solution for this synthesis is to use microreactor technology. A series of experiments carried out using a Doehlert experimental design provided access to a mathematical model describing, for an initial fixed composition ratio [NH4+]0/[OCl-]0, the evolution of the yield as a function of temperature, the flow rate of the reagents and the number of sodium hydroxide equivalent. Thus, the optimal conditions for the synthesis of stoichiometric chloramine using microreactor technology were established.

\section{Response to Reviewers:}

Please find enclosed my answer to your questions:

(a) The experimental design method makes it possible to reduce the number of experiments when there are several parameters to be studied at the same time (here: $\mathrm{T}$, eq $\mathrm{NaOH}$, flow rate), in order to determine the best conditions for the best result as quickly as possible. This is the advantage of a predictive model.

This method is mainly used in industry to quickly determine the most relevant experiments to be carried out to optimize the conditions of a synthesis.

b) The microreactor technique using "microchannels" involves working under pressure to compensate the pressure drop due to the small diameter of the channels. Therefore, it is not strictly speaking a pressurized synthesis in a conventional reactor since it is not a conventional reactor. The pressure conditions in a microreactor finally allow us to approach the atmospheric pressure conditions in a conventional reactor.

To clarify this point, I have added an additional explanation sentence in the paragraph " limits of microreactor" (highlighting of the added text). 
I hope you will be satisfied with these answers.

Yours sincerely

Powered by Editorial Manager ${ }^{\circledR}$ and ProduXion Manager ${ }^{\circledR}$ from Aries Systems Corporation 


\title{
A new strategy for the synthesis of monomethylhydrazine using the Raschig process
}

\section{2 - Continuous synthesis of stoichiometric monochloramine using the microreactor technology}

\author{
D.M. Le, A. J. Bougrine, O. Duclos, V. Pasquet*, H. Delalu \\ Université Claude Bernard Lyon 1 \\ Laboratoire Hydrazines et Composés Energétiques Polyazotés - UMR 5278 \\ UCBL/CNRS/CNES/ArianeGroup \\ Bâtiment Raulin, 2 rue Victor Grignard, F-69622 Villeurbanne Cedex, France \\ tel. +334 724485 47, fax. +33472431291 \\ e-mail: veronique.pasquet@univ-lyon1.fr
}

\begin{abstract}
After having established in a previous paper that the synthesis of stoichiometric monochloramine is hazardous due to the high exothermicity of the reaction and the risk of decomposition of the solution from $36^{\circ} \mathrm{C}$, it appeared that the best solution for this synthesis is to use microreactor technology. A series of experiments carried out using a Doehlert experimental design provided access to a mathematical model describing, for an initial fixed composition ratio $\left[\mathrm{NH}^{+}\right]_{0} /[\mathrm{OCl}]_{0}$, the evolution of the yield as a function of temperature, the flow rate of the reagents and the number of sodium hydroxide equivalent. Thus, the optimal conditions for the synthesis of stoichiometric chloramine using microreactor technology were established.
\end{abstract}




\section{Introduction}

In the first part of our study already published $^{(1)}$, we concluded that given the instability of stoichiometric monochloramine solutions from $36{ }^{\circ} \mathrm{C}$ and in order to control heat exchanges, the microreactor technology will be the best choice to avoid any sudden degradation leading to the formation of nitrogen chloride $\mathrm{NCl}_{3}$. Thus, this second part studies the optimal conditions for the synthesis of stoichiometric chloramine using microreactor technology.

The development of miniaturized systems, known as "microsystems", has progressed considerably over the past twenty years ${ }^{(2,3)}$. More recently, these technologies have stimulated the development of microreactors in the field of process engineering and industries with chemical-related problems. With regard to the synthesis of chloramine under stoichiometric conditions, we know that it is highly exothermic and can easily lead to the formation of explosive by-products $\left(\mathrm{NCl}_{3}\right)$. Continuous synthesis with current laboratory equipment therefore presents significant risks. For this reason, glass microreactors were used. They will permit the study of this continuous synthesis by limiting the exothermicity of the reaction.

\section{Advantages and limitations}

\section{Advantages of microreactors}

A fundamental property of the microreactor is the high value of the ratio between its surface area and its volume (specific surface area). For example, this value for the Corning microreactor pilot unit used in this study is $2500 \mathrm{~m}^{2} / \mathrm{m}^{3}$, with channels with a hydraulic diameter of $0.7 \mathrm{~mm}$. Wall phenomena are thus intensified. In particular, heat transfer is significantly increased compared to the other type of reactors ${ }^{(4)}$. Table 1 shows, for example, a comparison between different reactor types.

Table 1 
These transfer characteristics allow for better control and accuracy of operating conditions compared

\section{Limits of microreactors}

Microreactors are limited by the laws of hydrodynamics. Indeed, the miniaturization of the channels inevitably leads to an increase in pressure drop. In order to compensate this pressure drop, it is necessary to work under pressure to have a sufficient flow in the channels. On the laboratory microreactor unit, the pressure is in the range of 7 to 10 bar for flows ranging from 80 to $120 \mathrm{~mL} \mathrm{~min}^{-}$

${ }^{1}$. On the other hand, glass remains very fragile in the face of mechanical stresses and strains. Synthesis is therefore limited to pressures below 20 bar with a maximum total flow rate of $200 \mathrm{~mL} \mathrm{~min}^{-1}$.

For continuous use of microreactors at the industrial level, the passage of reagents will lead to progressive fouling of the microchannels. They will therefore have a limited operating time, which will vary depending on the reagents used and the products formed during the synthesis. This pollution will 
therefore require a special cleaning protocol and, possibly in the long term, a replacement cycle for microreactor modules.

\section{Experimental part}

Corning microreactor:

The study of the continuous synthesis of stoichiometric monochloramine was performed on the Corning microreactor unit shown in Fig. 1.

The different elements of the Corning microreactor unit are as follows:

- 2 DTR microstructures: pre-cooling plates,

- 1 MFA microstructure: separation of the bleach supply into 4 streams,

- 1 MJ microstructure: reaction plate composed of 4 mixing zones.

For each structure, the experimental conditions are as follows:

DTR microstructure: $\mathrm{V}_{\text {reagent: }} 9 \mathrm{~mL}, \mathrm{~V}_{\text {heat exchange: }} 14 \mathrm{~mL}, \mathrm{~S}_{\text {exchange }}: 17500 \mathrm{~mm}^{2}, \Delta \mathrm{P}: 100 \mathrm{mbar}$

MFA microstructure: $V_{\text {reagent }}: 1 \mathrm{~mL}, V_{\text {heat exchange }}: 13 \mathrm{~mL}, \mathrm{~S}_{\text {exchange }}: 3000 \mathrm{~mm}{ }^{2}$

MJ microstructure: $V_{\text {reagent }}: 10 \mathrm{~mL}, V_{\text {heat exchange: }} 16 \mathrm{~mL}, S_{\text {exchange }}: 23500 \mathrm{~mm}^{2}, \Delta \mathrm{P}: 2500 \mathrm{mbar}$

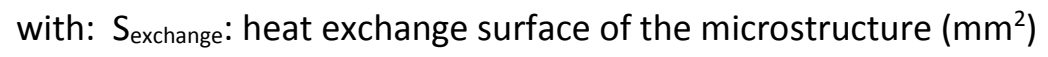

and $\Delta \mathrm{P}:$ pressure drop in the microstructure (mbar) (Pressure drops are measured with water at a flow rate of $100 \mathrm{~mL} \mathrm{~min}-1$ and at $20^{\circ} \mathrm{C}$ ).

The so-called "microreactor" part is composed of 4 glass modules. The 2 DTR modules are designed to pre-cool the reagents at different temperatures. The MFA module is a manifold that divides the bleach supply into 4 streams of equal flow rates. The MJ module is the reaction module with 4 mixing zones. 
It provides mixing and residence time actions at each injection point. The hydraulic diameter of the channels in the glass modules is $0.7 \mathrm{~mm}$ (manufacturer's data). The fluid flow regime in the microreactor unit is therefore laminar (Reynolds number is less than $10^{4}$ ).

In order to overcome the laminar regime, obstacles are created in the MJ microstructure plate (Fig. 2). These obstacles aim to break the flow and create a turbulent flow, which means better heat exchange and better mixing action.

Temperature control is provided by Lauda Integral T 2200 thermostats with $2.7 \mathrm{~kW}$ cryogenic power. They allow working in a temperature range from $-25^{\circ} \mathrm{C}$ to $120^{\circ} \mathrm{C}$. Reagent flow is controlled with two HNP Mikrosysteme Mzr-7259Ex gear pumps (PP1, PP2) and two ROTAMASS series 3 Coriolis mass flowmeters (FM1, FM2) supplied by Yokogawa. Sensors T1, T2, T3 and P1, P2, P3 are used to monitor the temperature and pressure of the fluids before and after reaction, as well as the system pressure drops as a function of the fluid flows.

\section{Chemical products:}

The permuted water used is city water purified by passing over an ion exchange resin. The inorganic salts and organic solvents used are of commercial purity (minimum 98\%) and are supplied by Acros Organics, Merck and Sigma-Aldrich. They were used without prior purification unless otherwise indicated. Aqueous solutions of sodium hypochlorite $\mathrm{NaOCl}$ and sodium hydroxide are supplied by Arkema (Jarrie Plant, Grenoble, France). The aqueous solution, measuring approximately 48 chlorometric degrees $\left(2.4 \mathrm{~mol} \mathrm{~L}^{-1}\right)$, is stored at $5{ }^{\circ} \mathrm{C}$ and systematically titrated before use.

Analytical methods:

lodometric dosage 
This method was used to determine the active chlorine concentrations of chloramine solutions and

hypochlorite ions. It is based on the oxidation of potassium iodide in acetic medium with titration of iodine released by a $0.1 \mathrm{M}$ sodium thiosulfate solution. The dosing reaction was monitored by potentiometry using a Metrohm 6.0451.100 combined platinum electrode.

\section{UV spectrometry}

The spectrophotometer used was an Agilent Cary 100 dual beam spectrophotometer equipped with the Cary WinUV data acquisition system. It allows a repetitive scanning of spectra between $180 \mathrm{~nm}$ and $900 \mathrm{~nm}$, programmable as a function of time, and measurements of optical density or its derivatives at a given wavelength. Measurements were made with $\mathrm{Hellma}^{\circledR}$ brand Suprasil ${ }^{\circledR}$ quartz cells, model 100-QS with a $10 \mathrm{~mm}$ optical path, to ensure optimal transmission of UV signals.

Continuous chloramine synthesis:

Between two manipulations, an isopropanol solution is injected into the device to ensure proper preservation of the pumps and glass microstructures. The rinsing solution used to purge and clean the installation is then a mixture of $25 \%$ water and $75 \%$ ethanol.

The reagent solutions $\left(\mathrm{NH}_{4} \mathrm{Cl}\right.$ solution and alkaline bleach solution) are previously prepared and cooled (generally $-5{ }^{\circ} \mathrm{C}$ ). Before each synthesis experiment, a water/ethanol solution is circulated at the desired flow rate to remove isopropanol from the pipes. Once the isopropanol has been purged, $\mathrm{NH}_{4} \mathrm{Cl}$ is introduced and then bleach. The synthesis is carried out, for all experiments, under a pressure of 11 bar. During normal operation, the steady state is reached after $10 \mathrm{~min}$. Samples are then taken punctually, every $15 \mathrm{~min}$, then analyzed by iodometric dosage and UV - Visible spectrometry. Measurements of temperature, fluid density and pressure are carried out online at different points in the installation in order to check that the synthesis is proceeding correctly and to make an immediate correction in the event of significant deviations from the instructions. $\mathrm{A} \mathrm{pH}$ monitoring at the end of the reaction module is necessary to avoid any drift of the monochloramine solutions. Indeed, a pH 
below 10 leads to the potential formation of highly explosive dichloramine $\mathrm{NHCl}_{2}$ and nitrogen trichloride $\mathrm{NCl}_{3}$.

At the end of the synthesis, the microreactor unit is rinsed with a water/ethanol solution for $30 \mathrm{~min}$, then an isopropanol solution is injected to preserve the pumps and microstructures.

\section{Results and discussion}

\section{Experimental design}

\section{Experimental design method}

Throughout this study on the Corning microreactor unit, many parameters that could influence the synthesis were investigated. The classical experimental methodology consists of setting the level of all but one of the variables and measuring the system response for different values of this variable, which implies carrying out a considerable number of experiments. For example, for 4 variables at 4 levels (i.e. using 4 different values for each variable), $4^{4}=256$ experiments must be performed. In order to avoid too many experiments, the design of experiments method was used.

Indeed, the designs of experiments are derived from mathematical and statistical methods applied to experimentation. The principle of this technique is to simultaneously vary the levels of one or more factors (discrete or continuous variables) in each experiment. This will significantly reduce the number of experiments to be carried out while increasing the number of factors studied, detecting the interactions between the different factors and a given response, i.e. a quantity used as a criterion, and allowing the results to be modelled. It should be noted that the main difference with the traditional method is that the design of experiments method allows the levels of all factors to be varied at the same time for each experiment, in a programmed and reasoned way ${ }^{(6)}$.

For this study, the factors studied were:

- the number of soda equivalent in bleach, 
- the temperature of the reagents (bleach and $\mathrm{NH}_{4} \mathrm{Cl}$ solution),

- the flow of fluids,

- the pressure.

\section{Complete factorial plan $\mathbf{2}^{3}$}

First, the most influential factors governing the system response will be sought rather than a specific relationship between factor variations and response variations. Previous studies in the laboratory have shown a significant influence of the amount of soda present in bleach on the monochloramine yield of the synthesis ${ }^{(7)}$. The influence of the remaining three factors (temperature, flow and pressure) therefore remains to be quantified.

To this end, a complete factorial design with these three factors (temperature, flow rate and pressure) was used, due to its simplicity and speed of implementation. This method consists of performing the experiments by varying the factors between a high (+) and low (-) level. In this case, this plan is rated $2^{3}$ ( 3 factors with 2 levels each), and therefore includes 8 experiments. The numerical values of the 2 levels of each of the 3 factors and the associated response (monochloramine yield) are given in Table 2.

Table 2

The mathematical model associated with the complete plan $2^{3}$ is as follows:

$y=a_{0}+a_{1} x_{1}+a_{2} x_{2}+a_{3} x_{3}+a_{12} x_{1} x_{2}+a_{13} X_{1} X_{3}+a_{23} x_{2} X_{3}+a_{123} X_{1} X_{2} X_{3}$

with :

$\mathrm{y}$ : the answer (in this case, the yield of the synthesis), $\mathrm{x}_{\mathrm{i}}$ : the abscissa of the experimental point for factor $\mathrm{i}$. Given the levels that the factors take, $\mathrm{xi}$ takes the value 1 for level (+) and -1 for level (-), 
$a_{0}$ : the value of the response at the center of the field of study,

$a_{i}$ : the effect of factor $i$,

$a_{i j:}$ : the interaction between factors $i$ and $j$ (the same for $a_{123}$ which is the interaction between the three factors).

The coefficients of the model can be determined using the following matrix equation:

$$
\left[\begin{array}{l}
Y_{1} \\
Y_{2} \\
Y_{3} \\
Y_{4} \\
Y_{5} \\
Y_{6} \\
Y_{7} \\
Y_{8}
\end{array}\right]=\left[\begin{array}{rrrrrrrr}
+1 & +1 & +1 & +1 & +1 & +1 & +1 & +1 \\
+1 & -1 & +1 & +1 & -1 & -1 & +1 & -1 \\
+1 & +1 & -1 & +1 & -1 & +1 & -1 & -1 \\
+1 & +1 & +1 & -1 & +1 & -1 & -1 & -1 \\
+1 & -1 & -1 & +1 & +1 & -1 & -1 & +1 \\
+1 & -1 & +1 & -1 & -1 & +1 & -1 & +1 \\
+1 & +1 & -1 & -1 & -1 & -1 & +1 & +1 \\
+1 & -1 & -1 & -1 & +1 & +1 & +1 & -1
\end{array}\right] \cdot\left[\begin{array}{l}
a_{0} \\
a_{1} \\
a_{2} \\
a_{3} \\
a_{12} \\
a_{13} \\
a_{23} \\
a_{123}
\end{array}\right]
$$

then, in condensed form:

$y=x \cdot a$

with:

y: column matrix containing the eight answers,

$\mathbf{X}$ : effects calculation matrix. This matrix is composed of the values taken by the levels of different factors or the products of the coordinates of these factors ${ }^{(8)}$,

a: column matrix of the coefficients of the mathematical model. 
The values of the coefficients of the model are obtained by solving the matrix equation.

The effects of the different factors and their interactions are shown in Fig. 3.

To conclude, Fig. 3 shows that among these three factors, the most influential are, in order of importance, temperature and flow rate. Pressure is not a decisive parameter. These factors will now be addressed in a Doehlert plan.

\section{Composite plan of Doehlert}

The combination of all the results shows that the factors that have the most influence on the yield of monochloramine synthesis are: the amount of $\mathrm{NaOH}$ in bleach, temperature and fluid flow. Pressure has very little influence and is therefore now set for all experiments at 11 bar, a value similar to that of the installations at the industrial ArianeGroup site in Toulouse (France).

In order to study the effects of these three factors on performance, a Doehlert plan of experiments is established, because of its advantages over factor designs. The first advantage of Doehlert plan is that each factor now has several levels (instead of 2 as in factorial plan $2^{3}$ ). The number of levels for each factor is variable, which allows the operator flexibility to assign a large number of levels or not to a factor. In this study, the "number of soda equivalent" factor was chosen as the factor with the highest 
number of levels, in order to obtain the maximum information from the system. Finally, the representative points of the different experiments in the Doehlert plan are placed according to a geometric figure, which ensures that the experiments are regularly placed in the experimental space ${ }^{(8)}$. Fig. 4 shows, schematically, the distribution of experiments for a three-factor Doehlert plan.

The postulated mathematical model used with Doehlert plan is a second degree equation:

$$
y=a_{0}+a_{1} x_{1}+a_{2} x_{2}+a_{3} x_{3}+a_{12} x_{1} x_{2}+a_{13} x_{1} x_{3}+a_{23} x_{2} x_{3}+a_{11} x_{1}{ }^{2}+a_{22} x_{2}^{2}+a_{33} x_{3}^{2}
$$

with :

Y: the answer (in our case, the yield of the synthesis),

$X_{i}$ : the abscissa of the experimental point for factor $\mathrm{i}$ in coded coordinates (Table 3 ),

$a_{i}, a_{i j}$ : coefficient of the mathematical model.

The parameters of the plan experiments and the experimental results are shown in Table 3:

Table 3

In this case, the calculation matrix is a $15 \times 10$ matrix since there are 15 experiments and 10 coefficients in the mathematical model. These coefficients are calculated by the following formula ${ }^{(8)}$ :

with :

$$
\mathbf{a}=\left(\mathbf{X}^{\top} \cdot \mathbf{X}\right)^{-1} \cdot \mathbf{X}^{\top} \cdot \mathbf{Y}
$$

a: vector of coefficients,

$X:$ calculation matrix $\left(X^{\top}\right.$ : matrix transposed from $\left.X\right)$,

$\mathrm{y}$ : vector of experiment results.

After solving the matrix equation, the mathematical model is as follows:

$y=97.73-1.642 x_{1}-4.003 x_{2}-3.505 x_{3}+39.193 x_{1} x_{2}-12.883 x_{1} x_{3}-6.568 x_{2} x_{3}+0.030 x_{1}^{2}-40.578 x_{2}^{2}-$ $4.770 \mathrm{x}_{3}^{2}$

Table 4 shows the experimental and modelled results for each experiment performed. 
The overall quality of the adjusted mathematical model is assessed using the following two statistical tools ${ }^{(9)}$ :

\section{- Fisher test}

This test aims to reject the hypothesis $\left(H_{0}\right)$ that the model does not describe the variation of the tests.

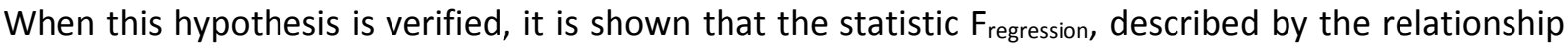
below, follows a Fisher law with, $v_{X}$ and $\nu_{R}$ degrees of freedom ${ }^{(10)} \cdot v_{X}$ and $\nu_{R}$ are the numbers of degrees of freedom associated, respectively, with the sum of the squares of deviations from the regression average value and the sum of the squares of the residues.

$$
F_{\text {régreasion }}=\frac{\sum\left(\hat{\mathrm{Y}}_{\mathrm{i}}-\overline{\mathrm{Y}}\right)^{2}}{\sum\left(\mathrm{Y}_{\mathrm{i}}-\hat{\mathrm{Y}}_{\mathrm{i}}\right)^{2}}
$$

with:

$\bar{y}$ : average value of experimental results,

$\mathrm{y}_{\mathrm{i}}$ : experimental value for experiment number $\mathrm{i}$,

$\hat{y}_{\mathrm{i}}:$ value calculated from the model for experiment number $\mathrm{i}$.

Thus, this hypothesis is rejected with a probability $\alpha$ if

$$
F_{\text {regression }}>\mathrm{F}\left(\alpha ; v_{\mathrm{X}} ; v_{\mathrm{R}}\right)
$$

Here $F\left(\alpha ; \nu_{X} ; \nu_{R}\right)$ is the $(1-\alpha)$ quantile of a Fisher law with $v_{X}$ and $\nu_{R}$ degrees of freedom.

\section{- Coefficient of determination $R^{2}$ of the multilinear regression}

This coefficient is defined by the ratio of the dispersion of the results, explained by the model, to the total dispersion of the results: 
When $R^{2}=1$, the estimations by the mathematical model coincide with the measurements, while for $R^{2}=0$, the data are not at all aligned. The value of $R^{2}$, in this case, is 0.98 , which means that $98 \%$ of the variation in the experiments is explained by the mathematical model.

The experimental results lead to Table 5.

According to Table 5, the statistic $F_{\text {regression }}$ satisfies the Fisher test with a 95\% confidence level. This mathematical model therefore makes it possible to describe the response of the experiments in a satisfactory way.

\section{- Canonical analysis}

The mathematical model associated with Doehlert plan is a second order polynomial model, so a canonical analysis can be performed. This technique allows to easily deduce the essential characteristics of the model such as: the coordinates of the stationary point of the model and the associated response, the type of the stationary point (maximum, minimum, minimax), the main axes as well as the variation of the model relative to each of these axes ${ }^{(11)}$. To do this, the mathematical model was written in the following matrix form:

$$
y=a_{0}+\mathbf{x}^{\top} \cdot b+\mathbf{x}^{\top} \cdot \mathbf{B} \cdot \mathbf{x}
$$


with

$$
x=\left[\begin{array}{l}
x_{1} \\
x_{2} \\
x_{3}
\end{array}\right]
$$

$$
b=\left[\begin{array}{l}
a_{1} \\
a_{2} \\
a_{3}
\end{array}\right]
$$$$
=\left[\begin{array}{c}
-2.67 \\
3.69 \\
0.11
\end{array}\right]
$$

$$
B=\left[\begin{array}{ccc}
a_{11} & 1 / 2 a_{12} & 1 / 2 a_{13} \\
1 / 2 a_{12} & a_{22} & 1 / 2 a_{23} \\
1 / 2 a_{13} & 1 / 2 a_{23} & a_{33}
\end{array}\right]=\left[\begin{array}{ccc}
-0.47 & 9.84 & -3.92 \\
9.84 & -26.88 & -4.92 \\
-3.92 & -4.92 & -6.37
\end{array}\right]
$$

The matrix equation of the second degree model implicitly implies the existence of a so-called stationary point $\mathrm{S}$ (unique) for which the partial derivatives of the response with respect to variables $x_{i}$ are all zero. Thus:

$$
\frac{\partial y}{\partial \mathbf{x}}=0
$$

wich gives :

$$
\mathbf{x}_{S}=-\frac{1}{2} \mathbf{B}^{-1} \mathbf{b} \quad \text { and } \quad y_{S}=a_{0}-\frac{1}{4} \mathbf{b}^{T} \mathbf{B}^{-1} \mathbf{b}
$$

The stationary point is therefore:

$$
\mathrm{x}_{\mathrm{S}}=\left[\begin{array}{l}
0.48 \\
-0.21 \\
-0.64
\end{array}\right]=\left[\begin{array}{l}
\text { temperature }=15.7^{\circ} \mathrm{C} \\
\text { eq } \mathrm{NaOH}=0.21 \text { equivalent } \\
\text { flow rate }=44 \mathrm{~mL} \mathrm{~min}^{-1}
\end{array}\right] \quad \text { and } \mathrm{y}_{\mathrm{s}}=102.49
$$


A significant simplification can be achieved if the mathematical model is seen from a particular benchmark, translated and returned relative to the original benchmark (factor benchmark $\mathrm{x}_{\mathrm{i}}$ ). To do this, first of all, the axes of the original reference frame will be rotated around its origin. The purpose of this operation is to remove the interaction monomas between factors $\mathrm{x}_{\mathrm{i}}$. Once the rotation is completed, a translation will allow the removal of the first order terms from the polynomial model. Fig. 5 shows, for example, the position of the axes during these operations.

The objective of this transformation is to bring the model back to the following canonical form:

$$
y=y_{s}+\lambda_{1} \cdot z_{1}{ }^{2}+\lambda_{2} \cdot z_{2}{ }^{2}+\lambda_{3} \cdot z_{3}{ }^{2}
$$

with

$y$ : the response of the model (in this case, the yield of the synthesis),

$z_{i}$ : the $i^{\text {th }}$ coordinates of the experimental point in the final reference frame,

$\mathrm{y}_{\mathrm{s}}$ : the response at the stationary point $\mathrm{S}$,

$\lambda_{i}$ : the eigenvalues of matrix $B$.

To do this, matrix B must be transformed into a diagonal matrix. The notions of vectors and eigenvalues then come into play. We will note $\Theta$ the diagonal matrix whose element $\Theta_{i i}$ is the eigenvalue $\lambda_{i}$ of $B$.

$$
\Theta=\left[\begin{array}{ccc}
\lambda_{1} & 0 & 0 \\
0 & \lambda_{2} & 0 \\
0 & 0 & \lambda_{3}
\end{array}\right]
$$

In the final reference frame, the mathematical model will therefore be written in the following matrix form: 
Similarly, the matrix is defined by $M$ for which the $\mathrm{i}^{\text {th }}$ column is the $\mathrm{i}^{\text {th }}$ eigenvector of $\mathrm{B}$. This matrix $\mathrm{M}$ plays an important role since it allows the passage of the coordinates of the experimental point in the original reference frame $x=\left[x_{1}, x_{2}, x_{3}\right]^{\top}$ to the coordinates $z=\left[z_{1}, z_{2}, z_{3}\right]^{\top}$ in the final reference frame, and vice-versa:

$$
\begin{gathered}
\mathbf{z}=\mathbf{M}^{\top} \cdot\left(\mathbf{x}-\mathbf{x}_{\mathrm{s}}\right) \\
\mathbf{x}=\mathbf{x}_{\mathrm{S}}+\mathbf{M} \cdot \mathbf{z}
\end{gathered}
$$

The numerical results in the case of this mathematical model are presented:

$$
\boldsymbol{\Theta}=\left[\begin{array}{ccc}
-30.65 & 0 & 0 \\
0 & 5.25 & 0 \\
0 & 0 & -8.32
\end{array}\right]
$$

$$
\text { and } M=\left[\begin{array}{ccc}
0.2898 & -0.8464 & 0.4467 \\
-0.9461 & -0.3239 & -0.0001 \\
-0.1448 & 0.4226 & 0.8947
\end{array}\right]
$$

The unit vectors of the final reference frame, expressed in coordinates of the original reference frame with their associated eigenvalues, are:

$$
z_{1}=\left[\begin{array}{l}
0.2898 \\
-0.9461 \\
-0.1448
\end{array}\right] \quad z_{2}=\left[\begin{array}{l}
-0.8464 \\
-0.3239 \\
0.4226
\end{array}\right] \quad z_{3}=\left[\begin{array}{c}
0.4467 \\
-0.0001 \\
0.8947
\end{array}\right]
$$


The mathematical model is therefore written:

$y=98.09-48.502 . z_{1}^{2}+11.195 \cdot z_{2}^{2}-8.012 \cdot z_{3}^{2}$

2 eigenvalues are negative $\left(\lambda_{1}\right.$ and $\left.\lambda_{3}\right)$ and one eigenvalue is positive $\left(\lambda_{2}\right)$. The response y decreases on axes $z_{1}$ and $z_{3}$ with negative eigenvalues, but increases on axis $z_{2}$. The order of magnitude of the coefficients is also important. Indeed, for the same variation of the $z_{i}$ component of $z$, its effect will be all the greater if the corresponding coefficient $\left(\lambda_{i}\right)$ is great. On the other hand, this effect will be all the less important if its coefficient is small. Fig. 6 shows the effects of changes of the $z_{i}$ components.

Fig. 7 visually shows the relative positions of the unit vectors of the original (xi) and final (zi) landmarks.

The canonical analysis that has been carried out shows that to increase the response y (the yield of monochloramine synthesis), it is necessary to move away from the stationary point $S$ along the $z_{2}$ axis (whose associated eigenvalue $\lambda_{2}$ is positive). 2 points are considered: $P_{1}=[0,1,0]_{\text {marker } z}$ and $P_{2}=[0,-1$, $0]_{\text {marker z. }}$ These two points therefore correspond to the following two experimental conditions: 


$$
\begin{aligned}
& P_{1}=\left[\begin{array}{l}
-0.36 \\
-0.53 \\
-0.22
\end{array}\right]_{\text {marker } x}=\left[\begin{array}{l}
\text { temperature }=2.2{ }^{\circ} \mathrm{C} \\
\text { eq } \mathrm{NaOH}=0.13 \text { equivalent } \\
\text { flow rate }=54.6 \mathrm{~mL} \mathrm{~min}{ }^{-1}
\end{array}\right]
\end{aligned}
$$

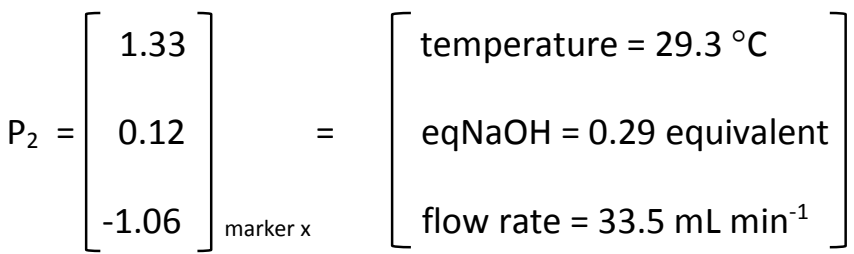

Point $P_{2}$ is rejected because of its high reagent temperature. The temperature of the chloramine solution at the outlet of the unit is therefore necessarily below $20^{\circ} \mathrm{C}$. This high temperature strongly favours the degradation of the solution and can potentially cause a sudden or even rapid degradation of the synthesis. For safety reasons, point $P_{1}$ is adopted as the optimal conditions for the synthesis of stoichiometric chloramine on the microreactor unit.

\section{Conclusion}

For the first time, the use of microreactors allows the continuous preparation of stoichiometric monochloramine. Under the experimental conditions studied, the study of the synthesis of $\mathrm{NH}_{2} \mathrm{Cl}$ on the microreactor pilot unit allows to conclude that:

- the total flow rate has very little influence on reaction performance, - two parameters have a decisive influence on the reaction performance, namely the temperature and the number of sodium equivalent in bleach,

- an excess of sodium hydroxide gradually leads to a drop in the initially almost quantitative yield from $97 \%$ to $84 \%$. This is due to a substitution reaction of $\mathrm{Cl}^{-}$by $\mathrm{OH}^{-}$with transient formation of 
hydroxylamine. The number of sodium equivalent does not exceed 0.25 to obtain a quasi-quantitative yield.

The lower bound of number of sodium equivalent is strongly influenced by temperature. Generally speaking, if the number of sodium equivalent is equal to 0.05 , a lower yield at $86 \%\left(T=0^{\circ} \mathrm{C}\right)$ is observed which is aggravated by a rise in temperature.

The major safety parameter is the initial concentration of sodium hydroxide in bleach. Whatever the temperature, this parameter must, in all cases, be higher than the minimum of 0.1 , preferably for safety reasons at $0.2-0.25$. Under these conditions, it is possible to carry out the synthesis preferably between 0 and $24{ }^{\circ} \mathrm{C}$.

To ensure process safety, it would be necessary to continuously measure the $\mathrm{pH}$ of the chloramine solution downstream of the reactor and/or perform an online UV measurement to verify the conformity of the absorption spectrum.

After discussion with our industrial partner and taking into account process safety aspects, we therefore recommend the following conditions for the synthesis of stoichiometric chloramine:

Temperature: $0{ }^{\circ} \mathrm{C}$

$\mathrm{NaOH}$ equivalent: 0.20 eq

Reagent flow rate: $50 \mathrm{~mL} \mathrm{~min}{ }^{-1}$

Pressure: 11 bar. 


\section{Bibliography}

(1) Le D M, Bougrine A J, Pasquet V, Delalu H (2019) A new strategy for the synthesis of monomethylhydrazine using the Raschig process. 1. Study of the stability of monochloramine. React Kinet Catal 127(2): 757-773

(2) Ehrfeld W, Hessel V, Löwe H (2000) Microreactors - New Technology for Modern Chemistry. $1^{\text {st }}$ ed. Wiley, VCH. Mainz

(3) Hessel V, Hardt S, Löve H (2004) Chemical Micro Process Engineering - Fundamentals, Modelling and Reactions. Wiley, VCH. Mainz

(4) Aubin J, Xuereb C (2008) Microréacteurs pour l'industrie. Base documentaire : Développement de solvants alternatifs et intensification des procédés. Techniques de l'ingénieur. Ref: IN94

(5) Frank T (2008) Microreactors in Organic Synthesis and Catalysis. Wiley, VCH Weinheim

(6) Goupy J (2001) Introduction aux plans d'expériences. $2^{\text {nd }}$ ed. Dunod, Paris

(7) Delalu H, Duriche C, Berthet J, Le Gars P (2006) Process for the synthesis of monochloramine. US Patent 7045659.

(8) Goupy J (1999) Plans d'expériences pour surfaces de réponse. Dunod, Paris

(9) Kamoun A, Chaabouni M M, Ayedi H F (2011) Plans d'expériences et traitement de surface Méthodologie des surfaces de réponses. Base documentaire: Traitements de surface des métaux: contexte et gestion environnementale. Technique de l'ingénieur. Ref: M1429

(10) Dodge Y, Rousson V (2004) Analyse de régression appliquée. Dunod, Paris

(11) Brisset S, Gillon F, Vivier S, Brochet P (2001) Optimization with experimental design: an approach using Taguchi's methodology and finite element simulations. IEEE Transactions on magnetics 37(5): 3530-3533 
(12) Lavric E D (2008) Thermal performance of Corning glass microstructures. Conference: ECl International Conference on Heat Transfer and Fluid Flow in Microscale. Whistler 
Table 1: Comparison table of current exchange modules ${ }^{(12)}$

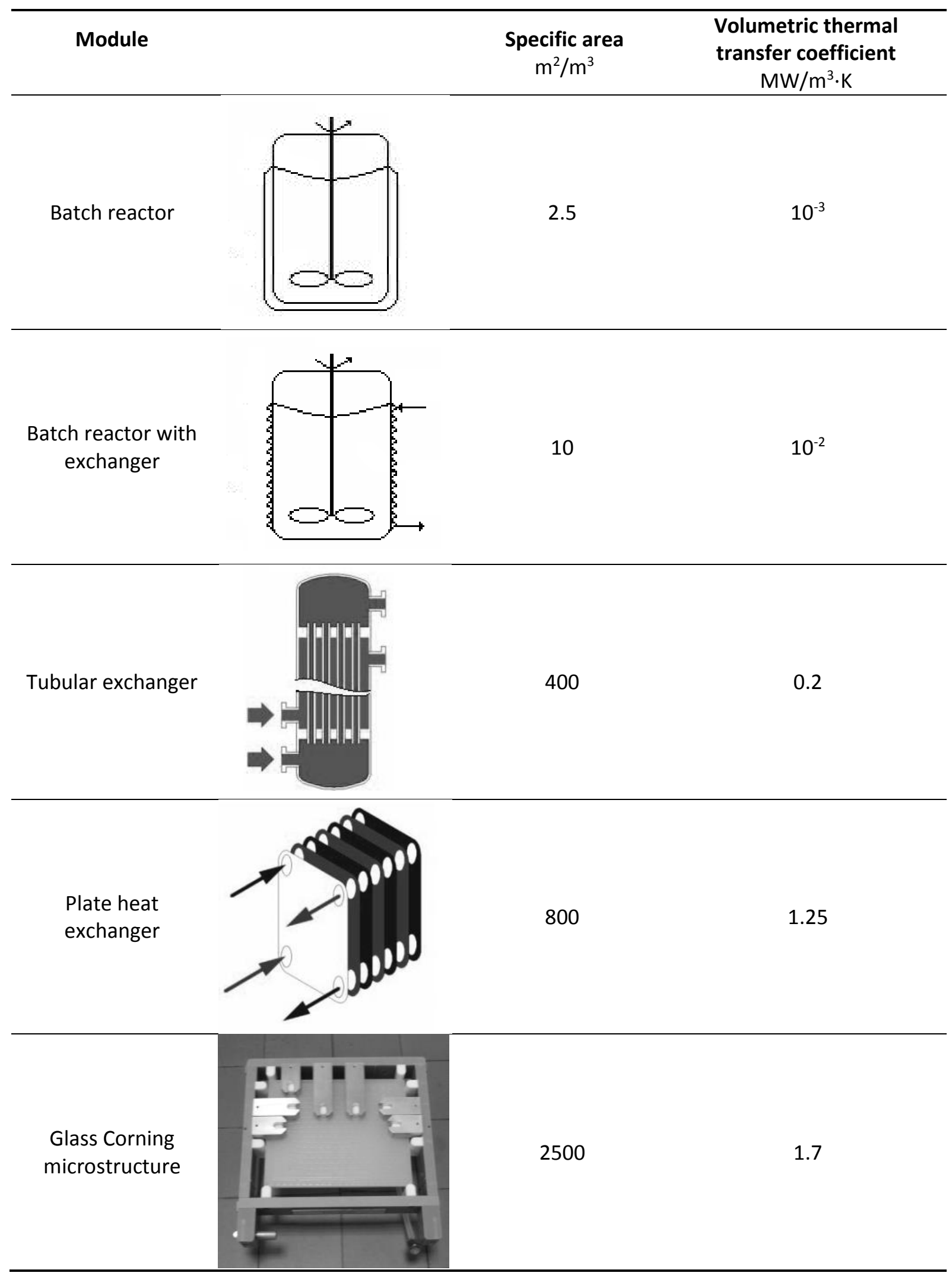


Table 2: Plan $2^{3}$ experience Matrix, summary of the experiments

\begin{tabular}{ccccc}
\hline $\begin{array}{c}\text { Experiment } \\
\text { number }\end{array}$ & $\begin{array}{c}\text { Factor 1 } \\
\text { Temperature }\end{array}$ & $\begin{array}{c}\text { Factor 2 } \\
\text { Flow rate }\end{array}$ & $\begin{array}{c}\text { Factor 3 } \\
\text { Pressure }\end{array}$ & $\begin{array}{c}\text { Answer } \\
\mathrm{NH}_{2} \mathrm{Cl} \text { Yield (\%) }\end{array}$ \\
\hline 1 & + & + & + & 86.9 \\
2 & - & + & + & 99.0 \\
3 & + & - & + & 86.0 \\
4 & + & + & - & 92.6 \\
5 & - & - & + & 99.5 \\
6 & - & + & - & 99.0 \\
7 & + & - & - & 83.2 \\
8 & - & - & - & 98.6 \\
\hline
\end{tabular}

\begin{tabular}{cccc}
\hline Effective values & ${ }^{\circ} \mathrm{C}$ & $\mathrm{mL} \mathrm{min}^{-1}$ & bar \\
\hline Level $(+)$ & 30 & 100 & 16 \\
Level $(-)$ & -10 & 30 & 7 \\
\hline
\end{tabular}


Table 3: Doehlert plan for three factors, summary of experimental tests

\begin{tabular}{|c|c|c|c|c|c|c|}
\hline \multirow[b]{2}{*}{$\begin{array}{l}\text { Experiment } \\
\text { number }\end{array}$} & \multicolumn{2}{|c|}{$\begin{array}{c}\text { Factor } 1 \\
\text { Temperature }\end{array}$} & \multicolumn{2}{|c|}{$\begin{array}{c}\text { Factor } 2 \\
\text { Soda equivalent }\end{array}$} & \multicolumn{2}{|c|}{$\begin{array}{l}\text { Factor } 3 \\
\text { Flow rate }\end{array}$} \\
\hline & $\begin{array}{l}\text { Encoded } \\
\text { value }\end{array}$ & $\begin{array}{c}\text { Real value } \\
{ }^{\circ} \mathrm{C}\end{array}$ & $\begin{array}{l}\text { Encoded } \\
\text { value }\end{array}$ & Real value & $\begin{array}{l}\text { Encoded } \\
\text { value }\end{array}$ & $\begin{array}{l}\text { Real value } \\
\mathrm{mL} \min ^{-1}\end{array}$ \\
\hline 1 & 0 & 8 & 0 & 0.26 & 0 & 60 \\
\hline 2 & 1 & 24 & 0 & 0.26 & 0 & 60 \\
\hline 3 & 0.5 & 16 & 0.866 & 0.47 & 0 & 60 \\
\hline 4 & -0.5 & 0 & -0.866 & 0.05 & 0 & 60 \\
\hline 5 & -1 & -8 & 0 & 0.26 & 0 & 60 \\
\hline 6 & -0.5 & 0 & -0.866 & 0.05 & 0 & 60 \\
\hline 7 & 0.5 & 16 & -0.866 & 0.05 & 0 & 60 \\
\hline 8 & 0 & 8 & 0 & 0.26 & 0 & 60 \\
\hline 9 & -0.5 & 0 & 0.289 & 0.33 & 0.816 & 80 \\
\hline 10 & 0 & 8 & -0.577 & 0.12 & 0.816 & 80 \\
\hline 11 & 0.5 & 16 & 0.289 & 0.33 & 0.816 & 80 \\
\hline 12 & -0.5 & 0 & -0.289 & 0.19 & -0.816 & 40 \\
\hline 13 & 0 & 8 & 0.577 & 0.40 & -0.816 & 40 \\
\hline 14 & 0.5 & 16 & -0.289 & 0.19 & -0.816 & 40 \\
\hline 15 & 0 & 8 & 0 & 0.26 & 0 & 60 \\
\hline Level $\quad-1$ & & -8 & & 0.02 & & 35 \\
\hline Level & & 8 & & 0.26 & & 60 \\
\hline Level +1 & & 24 & & 0.50 & & 85 \\
\hline
\end{tabular}

N. B. $: 0.866=\frac{\sqrt{3}}{2} ; 0.816=\frac{\sqrt{2}}{\sqrt{3}} ; 0.577=\frac{1}{\sqrt{3}} ; 0.289=\frac{1}{2 \sqrt{3}}$ 
Table 4: Results of the Doehlert plan experiments

\begin{tabular}{ccc}
\hline $\begin{array}{c}\text { Experiment } \\
\text { number }\end{array}$ & $\begin{array}{c}\text { Experimental } \\
\text { yield } \\
(\%)\end{array}$ & $\begin{array}{c}\text { Modelled yield } \\
(\%)\end{array}$ \\
\hline 1 & 97.73 & 97.73 \\
2 & 99.96 & 99.40 \\
3 & 83.38 & 81.63 \\
4 & 84.19 & 86.92 \\
5 & 95.56 & 96.12 \\
6 & 87.9 & 86.92 \\
7 & 54.62 & 54.62 \\
8 & 97.73 & 97.73 \\
9 & 84.95 & 84.39 \\
10 & 85.32 & 83.57 \\
11 & 84.52 & 86.83 \\
12 & 95.53 & 93.22 \\
13 & 82.92 & 84.67 \\
14 & 93.51 & 94.07 \\
15 & 97.73 & 97.73 \\
\hline
\end{tabular}


Table 5: Fisher test for regression

1

2

\begin{tabular}{lcclccc}
\hline $\begin{array}{l}\text { Source of } \\
\text { variation }\end{array}$ & $\begin{array}{c}\text { Degree of } \\
\text { liberty }\end{array}$ & $\begin{array}{c}\text { Sum of the } \\
\text { squares }\end{array}$ & Expression & Fregression & $F(0.95 ; 9 ; 5)$ & $R^{2}$ \\
\hline Total & $15-1$ & 1773.70 & $\sum\left(Y_{i}-\bar{Y}\right)^{2}$ & & & \\
Regression & $10-1$ & 1744.20 & $\sum\left(\hat{Y}_{i}-\bar{Y}\right)^{2}$ & 59 & 4.772 & 0.98 \\
residual & $15-10$ & 29.52 & $\sum\left(y_{i}-\hat{Y}_{i}\right)^{2}$ & & & \\
\hline
\end{tabular}


Fig. 1: Diagram of the Corning microreactor pilot unit

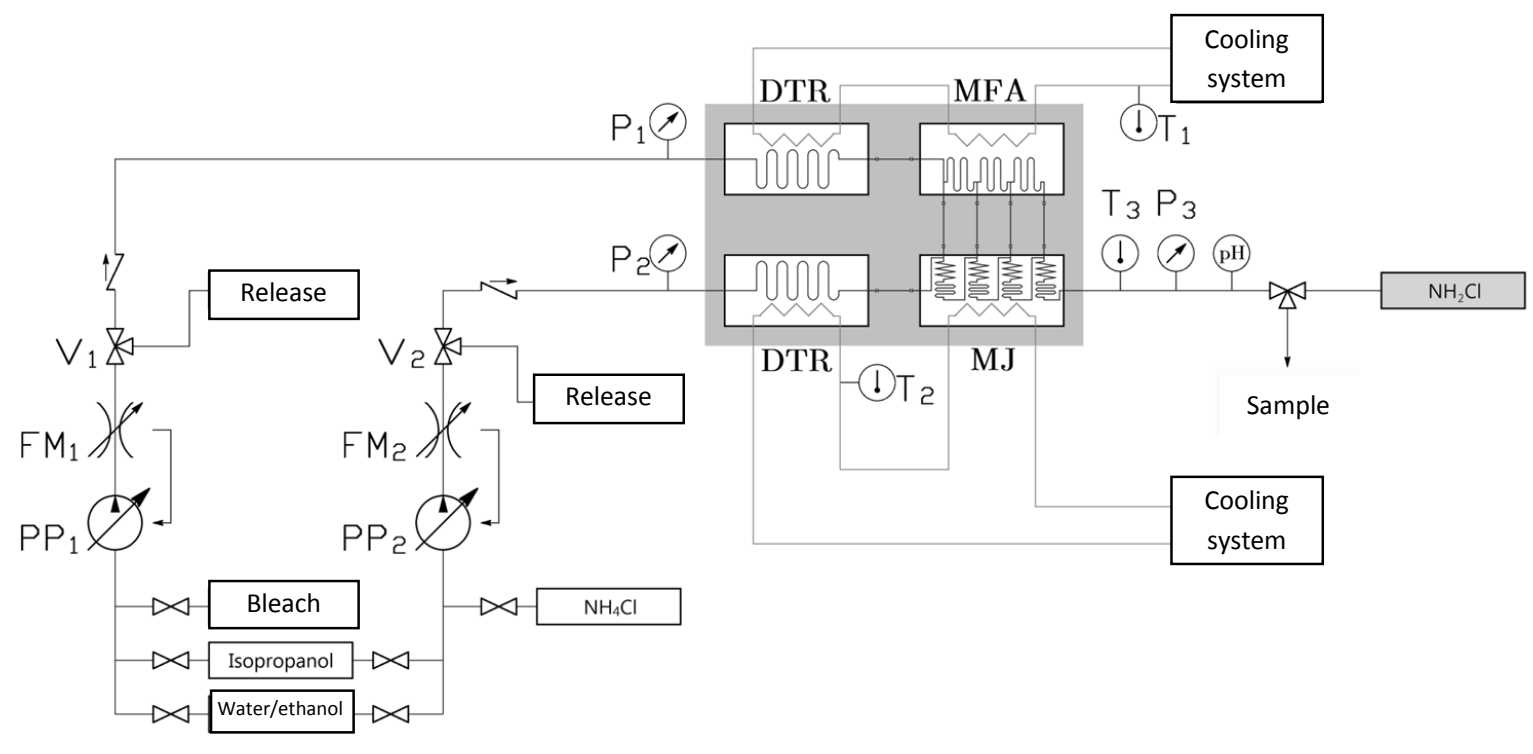


Fig. 2: Structure created in the MJ microstructure unit to break the flow

1

2

3

4

5

6

7

8

9 1 temperature-

1 epntrolled fluid

12 reagents

13

14

15

16

17

18

19

20

21

22

23

24

25

26

27

28

29

30

31

32

33

34

35

36

37

38

39

40

41

42

43

44

45

46

47

48

49

50

51

52

53

54

55

56

57

58

59

60

61

62

63

64

65

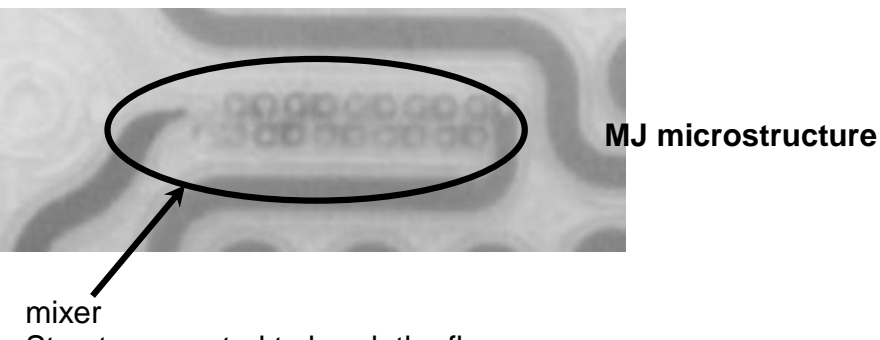

Structure created to break the flow

$\square$ Turbulent flow 
Fig. 3: Coefficients of the mathematical model of the plan $2^{3}$

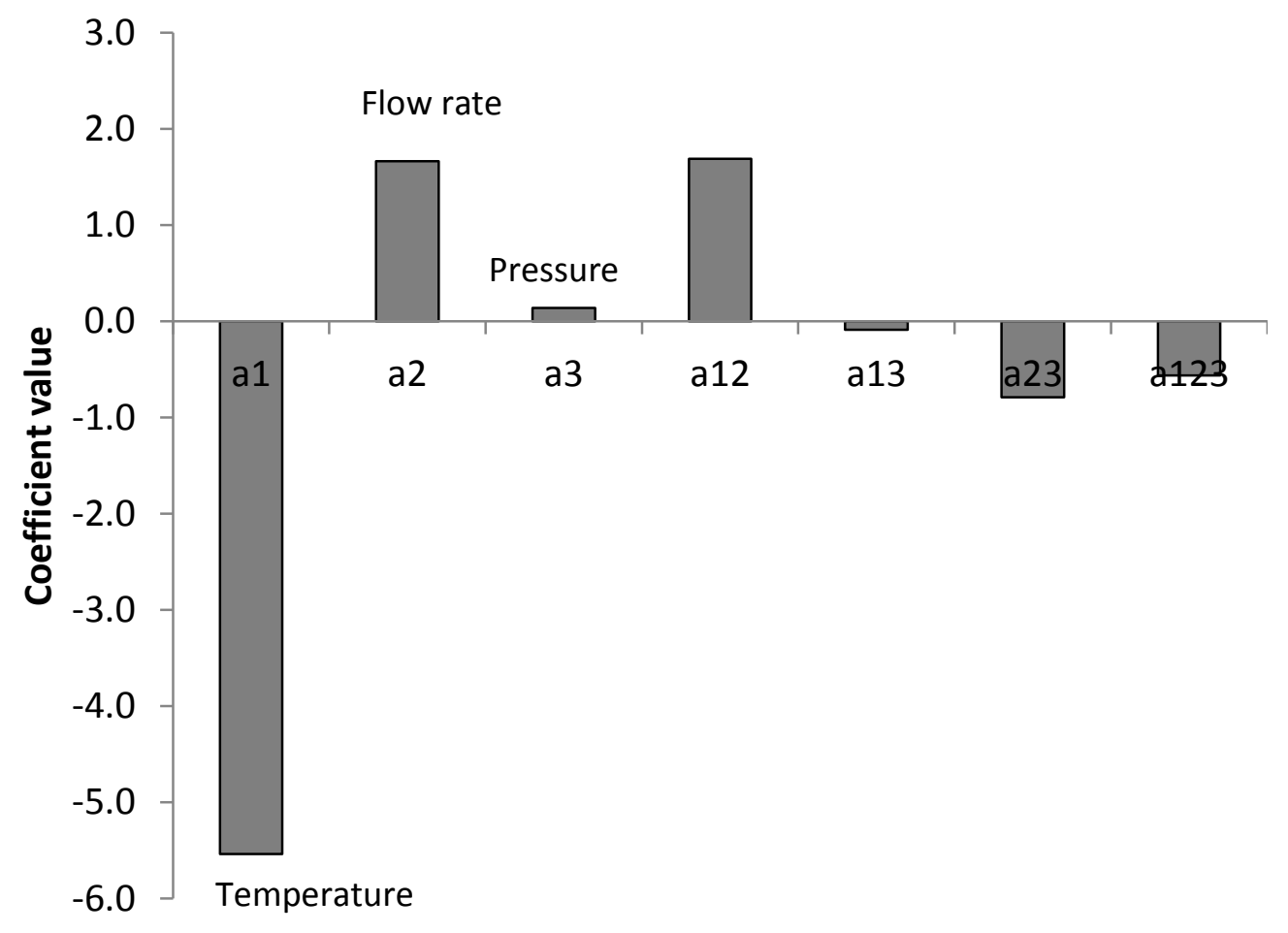


Fig. 4: Arrangement of Doehlert plan points for 3 factors

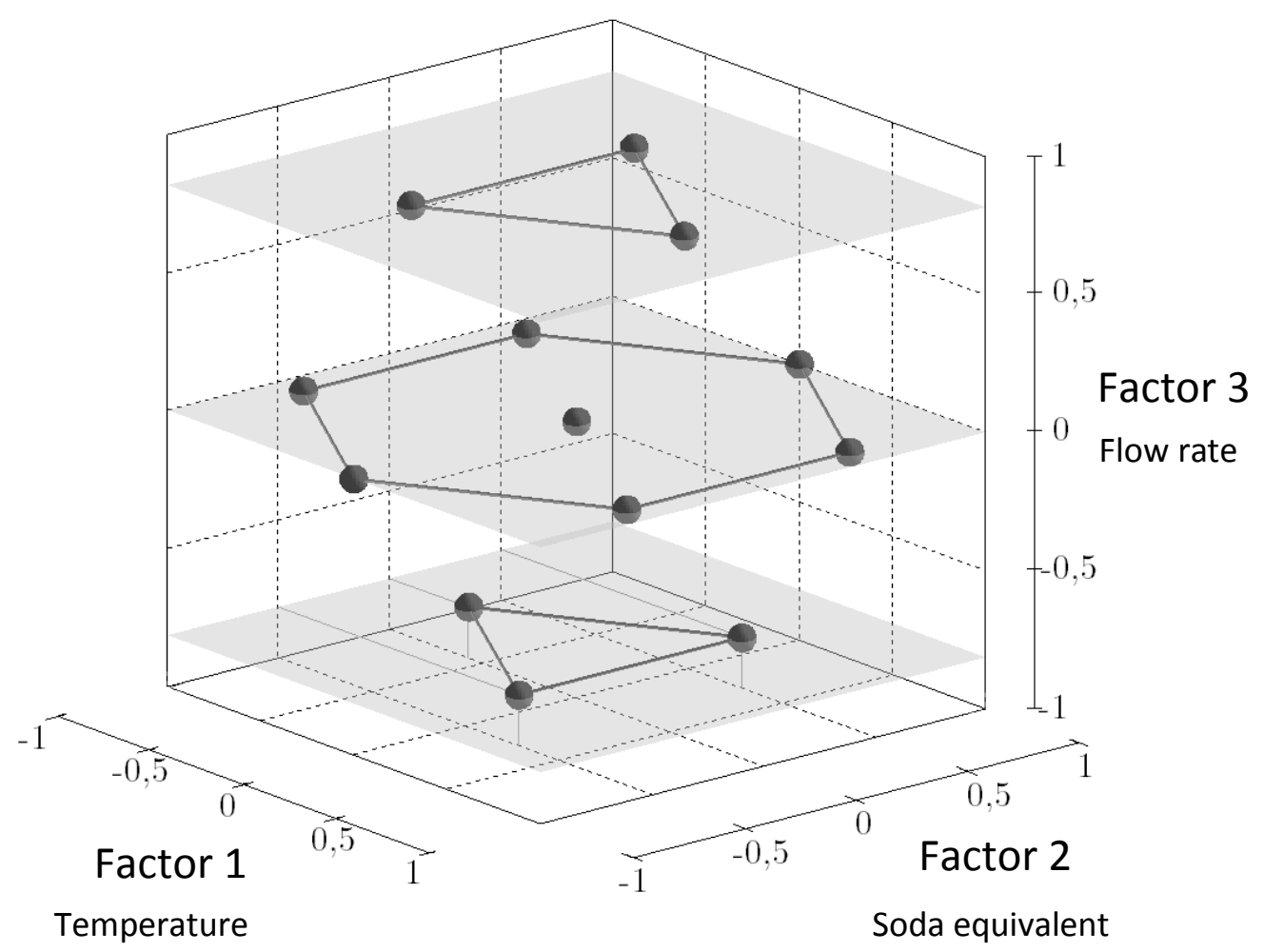


Fig. 5: Position of the axes during the transformation operations. (a): original position (original axes) ;

1

(b): positions of the axes during rotation ; (c): position of the axes during translation

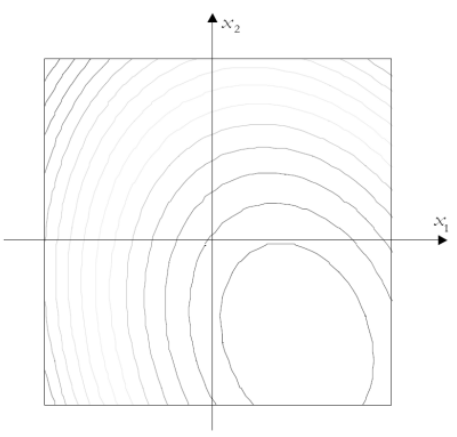

(a)

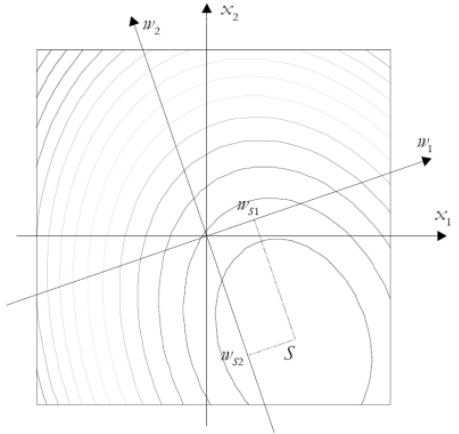

(b)

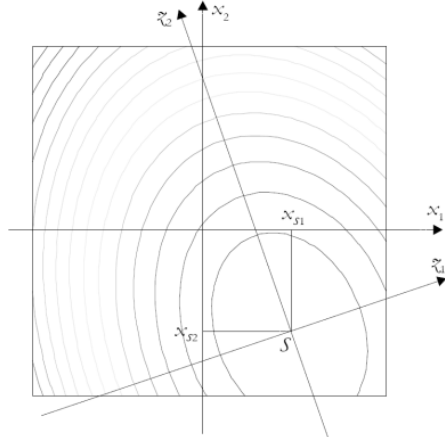

(c) 
Fig. 6: Variations of the response $y$ according to the axes $z_{i}$

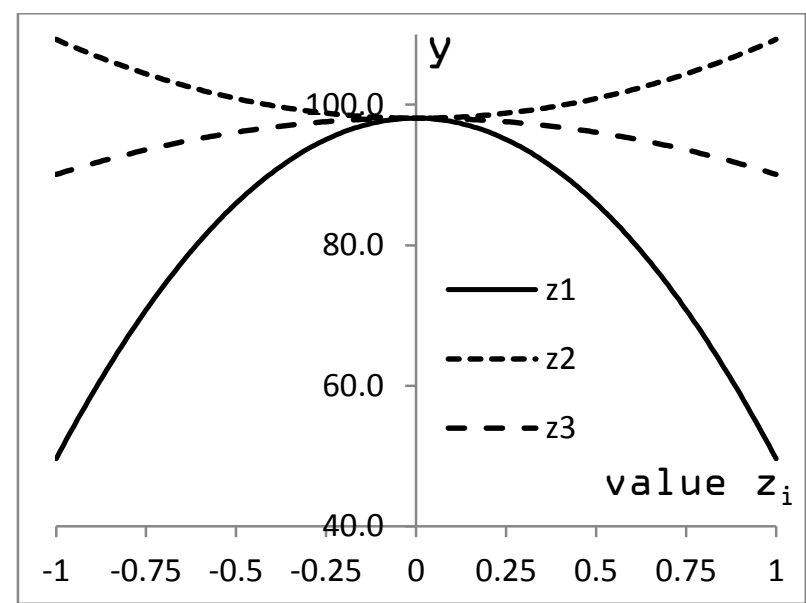


Fig. 7: Relative positions of the unit vectors of the original and final landmarks (views taken from different angles)
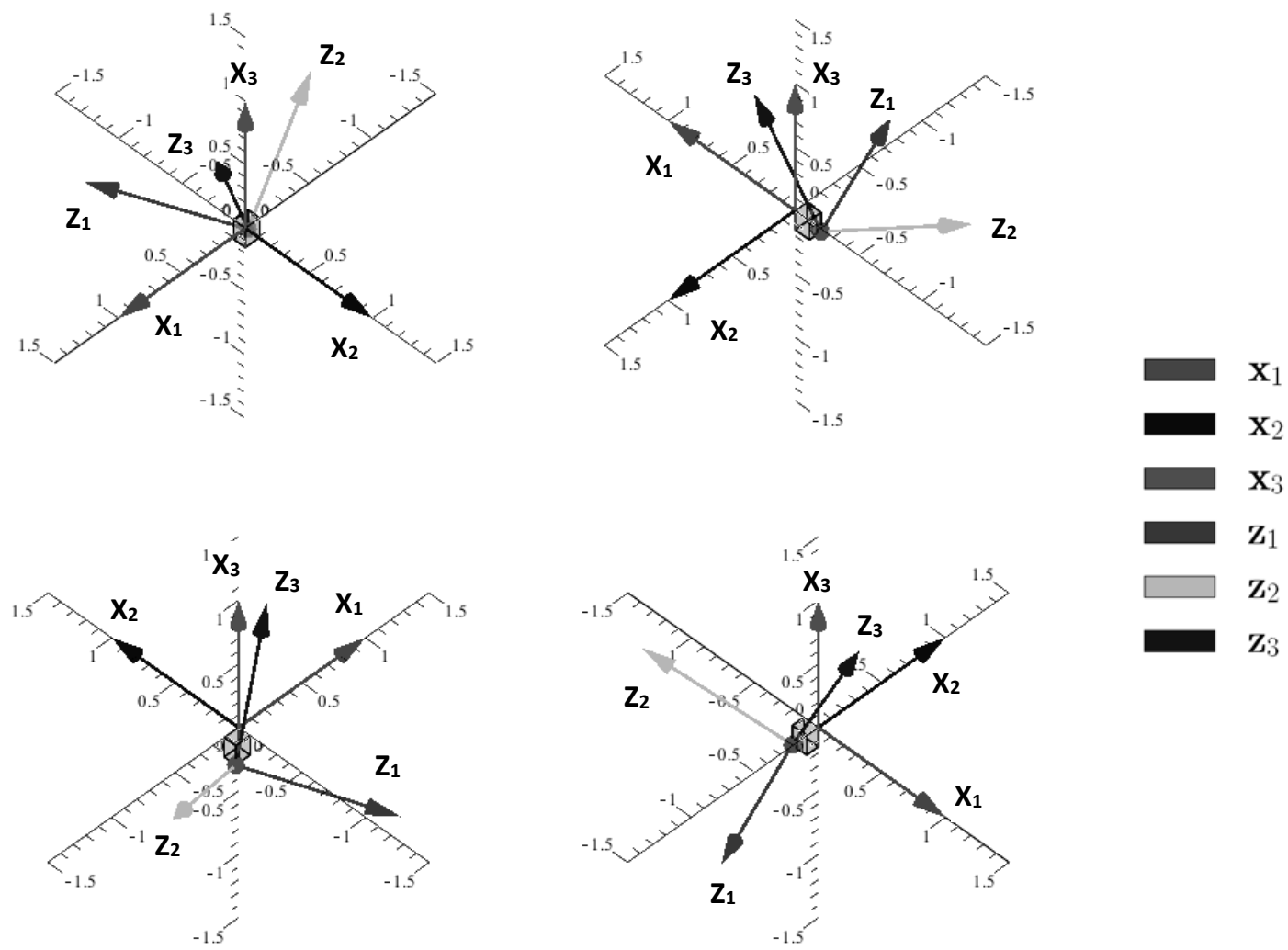

35

36

37 